\title{
Transportation Research into Practice: A Multi-Agency Government Perspective
}

\author{
Eric Neiderman, Federal Aviation Administration (Panel Chair) \\ Stephen M. Popkin, Volpe National Transportation Systems Center \\ Colleen Donovan, Federal Aviation Administration \\ Brian H. Philips, Federal Highway Administration \\ Sheryl Chappell, National Transportation Safety Board \\ Chris Monk, National Highway Traffic Safety Administration \\ Maura C. Lohrenz, Volpe National Transportation Systems Center (Panel Organizer)
}

\begin{abstract}
Safety is the top priority of the US Department of Transportation (DOT), with much of its \$1B of annual research and development budget focused on this priority. Each organization within DOT, and its oversight origination, the National Transportation Safety Board, have research programs that independently and jointly aim to impact and address these current and emerging safety issues, many of which are rooted in human factors causes. This panel will provide insight into understanding these issues, and how research was designed to deliver fieldable results that produced tangible safety benefits.
\end{abstract}

\section{INTRODUCTION ERIC NEIDERMAN, FAA}

The US Department of Transportation (DOT), along with its component Operating Administrations (OAs), is a major Research, Development, Test, and Evaluation (RDT\&E) funding source (\$1B annually) and an advocate for applied human factors. According to the US DOT Research Hub (http://ntlsearch.bts.gov/researchhub/index.do), at least 35 major DOT human factors initiatives are active at any one time. DOT human factors practitioners apply research and principles in the pursuit of improved transportation safety through effective acquisition, policy, and regulation. While there are numerous issues to research and investigate, only those impacting our agency's mission and strategic goals are supported and pursued.

Ultimately, the goal of each OA is to use its research funding to best meet its individual agency needs, within the framework of DOT priorities. The challenge for human factors practitioners, whether government, academic or private sector, is to interpret user/operator needs and translate them into meaningful requirements. These requirements are the first step in moving from successful research and testing to outcomes that impact the traveling public.

This panel provides context for human factors research to practice and impact, and focuses on key examples, both successful and otherwise, of processes used to transition the research into the field or regulation. This panel lays out challenges faced by human factors practitioners, the role of the basic and applied research community, the application of testing to effective design, and the impacts, intended or not, that human factors research has on transportation system safety within an evaluative, research-to-practice framework.

The following sections present examples of human factors research to practice for the Federal Highway Administration (FHWA), Federal Aviation Administration (FAA), National
Transportation Safety Board (NTSB), National Highway Traffic Safety Administration (NHTSA), and Research and Innovative Technology Administration (RITA).

\section{FEDERAL HIGHWAY ADMINISTRATION BRIAN PHILIPS, FHWA}

At the Federal Highway Administration (FHWA), we are focused on drastically reducing deaths and serious injuries on the nation's roadways. Consequently, the link between human factors research and practice is very important, since our research heavily influences both FHWA policy and gives practical results and guidelines that our State partners can implement in the field to improve roadway safety. At the FHWA Office of Safety Research and Development, we are focused on a number of research areas including driver distraction, roadway design, design of traffic control devices (including fixed signing and changeable message signs), and advanced technologies and warning systems.

FHWA addresses safety issues with human factors research in a number of different ways, using both applied approaches, as well as the more fundamental approaches, depending on the issue and research area. For example, in a more applied sense, we evaluate specific roadway and highway design elements for their suitability for drivers and other roadway users. This encompasses evaluating the design and safety of particular signing and traffic control devices. In a more basic (yet still very applied) research sense, we strive to improve our understanding of fundamental aspects of how drivers perceive, process, and respond to the roadway environment to inform better roadway design. For instance, this includes looking at eye glance behavior and visual scene complexity in the roadway environment. An example of the later is how we used eye glance behavior, visual background clutter, and sign recall measures to investigate the conspicuity of road signing. 


\section{Double Crossover Diamond Interchange}

This portion of the panel discussion will cover several research projects that have resulted in practical results that have been implemented in the field. The first project that will be discussed is the design and evaluation of a Double Crossover Diamond (DCD) freeway interchange. Although the DCD design has been successfully used in France for the last 30 years, it is a fairly new type of freeway interchange in the United States. Many conventional interchanges in urban areas are congested and experience high crash rates. The DCD design was shown in France to be safer and more efficient (less congested) than its conventional interchanges. The DCD design has a driver cross over from the right side of the road to the left side of the road and then back to combing left turning traffic with through traffic. Because of this novel design, one human factors concern was that drivers would drive down the wrong roadway section. Visualization simulations were created as well as driving scenarios in the FHWA highway driving simulator at Turner Fairbank Highway Research Center (TFHRC); see Figure 1. MoDOT engineers visited TFHRC to virtually drive through the simulated DCD; we also had other participants drive through the DCD, and no one went down the wrong road.

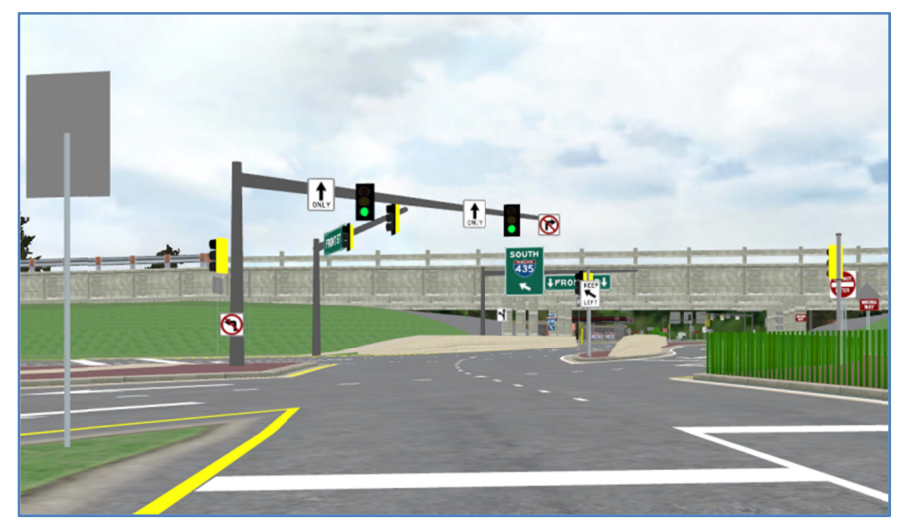

Figure 1. Image of the DCD driving scenario.

This human factors visualization and testing in the driving simulator helped to relieve safety concerns about the new design, and the associated videos helped facilitate public outreach efforts during public meetings. Missouri opened the first Double Crossover Diamond in Springfield Missouri in June 2009. Because fewer modifications to the existing roadway were needed than with a conventional interchange, the project was less expensive ( $\$ 3.2$ million vs. 10 million) and quicker to implement (6 months vs. $12-18$ months) as well as safer (50\% reduction in crashes) than a conventional interchange.

\section{Low-Cost Safety Improvements}

Another project that illustrates the successful transition of human factors research into practice is FHWA research in low cost safety improvements. Specifically, we investigated lowcost visibility enhancements for navigating rural horizontal curves at night. According to the Fatality Analysis Reporting System, approximately $17.4 \%$ of fatal crashes occur on horizontal curves. Of those crashes, about $42.2 \%$ occurred at night, so fatal crashes on rural curves at night represent an important safety problem.

This study involved a driving simulation experiment to evaluate two sets of alternative safety improvements for rural areas. The first set of improvements focused on enhancing curve visibility on rural two-lane undivided roads at night, to give drivers advanced detection of curves and thereby slow their speed prior to the curves. The second set of improvements was geared toward slowing traffic on rural twolane undivided roads in small towns during the day. The experiment was conducted at TFHRC's Highway Driving Simulator.

Several low-cost treatments yielded speed reductions: 1) post-mounted delineators (PMDs) enhanced by streaming light emitting diode (LED) lights slowed drivers the most (by an average of $9 \mathrm{mi} / \mathrm{h}, 2$ ) standard PMDs slowed drivers by $7-8$ $\mathrm{mi} / \mathrm{h}$, and 3 ) edge lines slowed drivers by $2 \mathrm{mi} / \mathrm{h}$. Slower speeds in the curves translated into fewer run-off-the-road crashes, injuries, and fatalities.

\section{Active Traffic Management and Information Distraction}

Two other on-going FHWA research projects are the Active Traffic Management (ATM) project and the Information as a Source of Distraction project. Both efforts are investigating the use of Changeable Message Signs (CMS) and what information can and should be displayed on CMS to help direct and guide drivers in a safe and efficient manner. The ATM project focuses on symbols and signs used for dynamic lane control and dynamic speed limits, while the distraction project focuses on evaluating different types of displayed information (including icons, road signs, and advertisements). Both of these projects will provide data and guidelines for the MUTCD (Manual for Uniform Traffic Control Devices) that will help the States design safer and more effective CMS and roadways.

The three projects described above show the strong link between the FHWA Human Factors research program and the application of research results into practice. The program supports policy creation and helps transportation engineers and safety specialists continue to make our roadways safer, and thereby decrease crashes, injuries, and fatalities.

\section{FEDERAL AVIATION ADMINISTRATION COLLEEN DONOVAN, FAA}

The Federal Aviation Administration (FAA) is comprised of approximately 47,000 individuals responsible for the safety of the public in the National Airspace System. Their mission is to provide the safest, most efficient aerospace system in the world. Human factors play a key role in the Agency's safety mission. The FAA funds approximately 20 million dollars in human factors-related research contracts per year in support of the Agency. Half of this funding supports FAA end users 
responsible for Air Traffic Control/Technical Operations, and the other half supports FAA end users within the FAA's Aviation Safety organization.

\section{Analysis of End Users}

The goal of this portion of the panel is to provide background and insight into the end users of FAA human factors research, in an effort to ensure that researchers funded by the FAA are meeting those needs, and that the Agency's efforts to transition "Research to Reality" are successful.

The panel will provide an overview of the types of FAA end users who have requested human factors research to support their needs within the Air Traffic Control/ Technical Operations Organization and the Aviation Safety Organization. Typically, FAA end users seek applied research results on which they can base their decisions to approve or not approve a specific system (e.g., ATC system or flight deck system). The goal is to base Agency decisions on empirical data, not opinion. Specifically, FAA end users rely on research results to determine minimum safety requirements for any system, procedure, maintenance operation, etc., under the Agency's purview. Thus, empirical evidence is used to make the safety case that the requirements are appropriate, which makes them defensible. It also ensures a level playing field for all applicants (airlines, avionics manufacturers, etc.) because all applicants are held to the same requirements.

\section{Selected Success Stories}

This portion of the panel will provide an overview of selected success stories in which the FAA transitioned from "Research to Reality" and directly used the results of the research. For example, a series of research projects on human factors issues of flight deck Global Positioning Systems (GPS) will be reviewed. Results of that research directly supported the FAA's current requirements for approving the GPS avionics proposed for approval by applicants such as Garmin. The research results were also used by FAA individuals who approve the operations and procedures associated with those systems. Finally, the human factors research resulted in FAA minimum requirements for pilot interfaces and safety issues with GPS receivers related to color, symbology, workload, etc. All of the research success stories presented will be traced from the initial research request, to analysis of end users' needs, to the final product delivered to the FAA, and the FAA's implementation of the results. Successful implementation of the research results directly impacts the FAA's mission to provide the safest, most efficient aerospace system in the world.

\section{NATIONAL TRANSPORTATION SAFETY BOARD SHERYL CHAPPELL, NTSB}

Following a tragic mid-air collision of an airliner and a small single-engine aircraft, the U.S. government mandated the installation of a traffic-alert and collision avoidance system (TCAS) in all airline aircraft. At that time, the FAA was still testing a TCAS prototype. The aviation industry and the FAA approached scientists with the National Aeronautics and Space Administration (NASA) to conduct research on the human factors associated with the design and operation of TCAS. Because it was a new system with no predecessor, there was a rare opportunity to shape the design around human performance and human error prevention. The human factors to be addressed included procedures, training, alerting, display symbology, system failures, and air traffic control operations. The research would drive the certification standards for the system worldwide.

Scientists tapped the vast body of human performance research in cockpit displays and controls to design the prototypes to be tested. Levels of alerting were created in compliance with the alerting standards of the US and international standards organizations. These standards are based on empirical human factors research and many years of successful implementation. The content of the voice alerts, which became the standard for TCAS, was the product of years of research on the intelligibility of aural alerts in the cockpit, especially voice. For example, in the initial design, voice warnings included "Do Not Climb" but the pilots often climbed in response, due to incorrect perception of the initial syllables and the negative statement of the command. NASA designed new aural alerts, which incorporated human factors research, and tested them with representative levels of ambient aircraft noise and air traffic communications. All pilot responses with the new aural alerts were in the correct direction.

Maneuver displays tested by the FAA produced insufficient and opposite-direction evasive maneuvers from airline flight crews during NASA simulations. Applying human factors principles, the NASA team was able to significantly improve reaction time and response accuracy with new display designs. The human factors of the traffic displays was the subject of an existing NASA research program, beginning with task analyses, followed by part-task and full-mission simulation studies. The findings from this program were applied to the TCAS traffic displays.

NASA human factors research also resulted in a significant change to the collision-avoidance logic in TCAS. Specifically, NASA research provided the data to show that a pilot response that required a reversal in the direction of the vertical avoidance maneuver was both possible and superior to not providing guidance in these rare situations.

Working closely with airline crews, TCAS procedures were designed to maximize the accuracy of the crew response, minimize crew response time, and reduce false alarms. The procedures included limiting the use of traffic display information for maneuvering, communication protocols with air traffic control, pilot-flying and pilot-monitoring responsibilities, and recommended modes for TCAS during specific operational conditions. 
A series of studies was conducted to examine the TCAS prototype, including changes to displays, controls, logic, and procedures in a full-mission simulation with current airline flight crews provided by the US airlines. These studies resulted in recommended crew procedures, changes to the collision avoidance logic, and visual and aural display standards for avoidance maneuver guidance and traffic displays. The TCAS design and procedures resulting from the NASA research findings were tested in airline revenue flight. Cockpit observers and digital data collection captured data on all aspects of the TCAS in normal line operations and its integration into the US National Airspace System.

The opportunity to perform the research was extended to the NASA Human Factors Team, but the research findings would not have become the international standards that they are, had the research team not been involved in the industry and government committees developing the standards. The participants in these committees included pilots, air traffic controllers, regulators, scientists, and manufacturers.

Dedicated follow-through by the committees and numerous scientific briefings by the NASA Team made the difference between an obscure government report and a recognized international standard. The team met their goal of developing a single standard for all cockpit implementations of TCAS to the greatest extent possible. The ensuing years of safe TCAS operation have demonstrated the appropriateness of the TCAS design standards.

\section{NATIONAL HIGHWAY TRAFFIC SAFETY ADMINISTRATION - CHRIS MONK, NHTSA}

The role of Human Factors research at the National Highway Traffic Safety Administration (NHTSA) has grown in recent years with so many technological advances in vehicle safety systems that rely heavily on the interaction with the driver. Increasingly, the importance of human factors for NHTSA's core mission of vehicle safety has been highlighted at the highest levels of the agency. The current research agenda focuses on topics such as driver distraction, connected vehicle technologies, crash warning interfaces (alerts), impairment detection systems, and auditory requirements for "quiet cars," to name a few. Translating research into practice is a crucial issue for NHTSA as it works to ensure the safety of the American driving public. The agency works to produce regulations when appropriate, while also working closely with industry and industry groups to fill research gaps when applicable. NHTSA produces guidelines and other research-based products intended to aid technology developers in ensuring their driver-vehicle interfaces meet established human factors criteria and guidance. These products are the embodiment of NHTSA's human factors research program, and demonstrate how NHTSA is pursues the translation of its research into the design of in-vehicle technologies. Three programs that exemplify these objectives are highlighted below.

\section{NHTSA Distraction Guidelines}

As part of NHTSA's overarching goal of reducing fatalities and injuries due to traffic crashes, the agency developed a comprehensive distraction plan in 2010 to help meet its long-term goal of eliminating crashes attributable to driver distraction. A key product of this plan is a set of voluntary Driver Distraction Guidelines (NHTSA Distraction Guidelines) to promote safety by discouraging the introduction of excessively distracting devices in vehicles. The first phase, titled the "Visual-Manual NHTSA Driver Distraction Guidelines for In-Vehicle Electronic Devices," covers original equipment in-vehicle device secondary tasks (e.g., communications, entertainment, information gathering, and navigation tasks not required to drive) performed by the driver through visual-manual means (i.e., the driver looking at a device, manipulating a device-related control with the driver's hand, and watching for visual feedback). The second phase will focus on portable and aftermarket devices, while the third and final phase will expand the guidelines to include auditory-vocal interfaces.

\section{Crash Warning Interface Metrics}

There was a role for government to produce standard metrics and evaluation protocols for Advanced Crash Warning System driver-vehicle interfaces (e.g., crash avoidance alerts). The objectives of the Crash Warning Interface Metrics (CWIM) program are to (1) demonstrate protocols for assessing the effectiveness and acceptability of Advanced Crash Warning System driver-vehicle interface systems, and (2) determine whether there are benefits to having consistency in ACWS characteristics across different vehicle models (or conversely, if there are adverse effects that could limit the overall effectiveness of the technologies in a broadly deployed marketplace scenario). (3) If there are benefits to consistency, a third objective is to specify the necessary consistent elements. This program is currently in its third phase, and NHTSA is coordinating with industry groups to ensure the final product is not only scientifically sound, but also usable by system developers.

\section{Human Factors for Connected Vehicles}

DOT is investing in the development of connected vehicle safety applications, which are designed to increase situational awareness and reduce or eliminate crashes through vehicle-to-vehicle (V2V) and vehicle-to-infrastructure (V2I) data transmission that supports driver advisories, driver warnings, and vehicle and/or infrastructure controls. These technologies may potentially address up to 82 percent of crash scenarios with unimpaired drivers, preventing tens of thousands of automobile crashes every year. Human Factors is a crucial part of the overall Connected Vehicle program. The program's objective is to assess, counteract, and ultimately eliminate possible driver distraction from technologies that enable wireless communication between 
vehicles. The program aims to research and implement technology-based solutions that could deter drivers from multi-tasking and reduce vehicular sources of distraction. An update on the multi-faceted human factors research program will be provided during this panel discussion.

\section{RESEARCH AND INNOVATIVE TECHNOLOGY ADMINISTRATION - STEPHEN POPKIN, VOLPE}

The US DOT re-established its Safety Council in 2009 to provide a forum where cross-cutting, significant safety issues could be addressed at the senior-most levels within the agency. This accomplishment in itself is an example of research to practice organizational design, such that 9 semiautonomous organizations were brought together to engage in addressing safety from a national transportation systems level, rather than separately addressing the parochial interest of a particular industry. Using an emergent program evaluation design the Council was developed to allow for cross-modal dialogue and action to address these concerns.

Specifically, the methodology involved included embedded evaluation and a stakeholder engagement strategy within an iterative design framework. Stakeholder interviews of the principals, including the Deputy Secretary of Transportation, were held, and through a collaborative process, the organization was founded, using a minimum specifications approach to keep it nimble and responsive to emerging needs (e.g., such as pipeline explosions or Deepwater Horizon, as examples of safety culture, staffing and training issues).

Ensuring sustainability, several levels of evaluation are performed concurrently, which influence the structure, operations, and ultimate success of the Council. This presentation will review the findings of this approach, including significant agency achievements to process improvement that reflects the emerging, and continually developing and maturing nature and role of this body.

With regard to output and outcomes on transportation safety human factors issues, this forum has developed five work areas over the past three years, each of which has a significant human factors component. Those areas are safety culture, operator fatigue, safety management systems, safety data use, and cyber security.

\section{SUMMARY MAURA LOHRENZ, VOLPE}

Human factors research in DOT profoundly impacts the safety of the traveling public. This is a tremendous responsibility that we take very seriously. This sense of accountability can also inspire innovative solutions to often complex transportation problems.

This panel presented several successful cross-agency examples of human factors research to practice. The hope is that lessons learned from these examples might help other human factors practitioners design, execute, and transition their own research into practical results that can be fielded and implemented. The panel is comprised of members of the DOT Human Factors Coordinating Committee (HFCC). The HFCC was established to enhance awareness, understanding, application, and evaluation of human factors in transportation. In an effort to facilitate human factors research-to-practice, the HFCC reaches out to academia and other government agencies to share ideas and encourage human factors researchers to consider DOT safety priorities when proposing new work.

A common theme among the presented success stories is close coordination and communication among various stakeholders, including 1) policy makers who decide which research is relevant and timely, 2) human factors practitioners who perform the research, 3) transportation engineers who implement solutions in the field, 4) manufacturers of resulting products, and 5) end-users of the final products. The process is often iterative, such that fielded solutions answer an original problem but expose other issues to be researched, as in the case of TCAS.

For several FHWA examples, successful research-topractice involved proving whether or not a lower-cost solution to a transportation problem would also be safer. Therefore, the research results were expected to impact both safety and economics, which is a powerful motivator for putting results into action. The FHWA also cultivates strong ties among their human factors researchers, transportation engineers and policy makers, which reinforces the researchto-practice paradigm.

For the FAA, research-to-practice often starts with the initial decision to embark on a research project. Research is funded only if there is good reason to believe the results will help agency decision-makers approve or disprove a new system or modifications to an existing system. Human factors researchers are recruited for skills and experience that will help answer that question.

Finally, the DOT Safety Council is a model of coordination and cooperation among various organizations, encouraging and supporting research-to-practice across DOT. The council helps focus DOT human factors research efforts on key work areas of primary interest to DOT safety concerns. 